\title{
Docosapentaenoic Acid n-6
}

National Cancer Institute

\section{Source}

National Cancer Institute. Docosapentaenoic Acid n-6. NCI Thesaurus. Code C68348.

A polyunsaturated very long-chain fatty acid with a 22-carbon backbone and 5 double bonds, orig inating from the 6th, 9th, 12th, 15th and 18th positions from the methyl end. 